Abstract Second-generation interferometric gravitational wave detectors require high-power lasers with approximately $200 \mathrm{~W}$ of output power in a linear polarized, single-frequency, fundamental-mode laser beam. Furthermore very high temporal and spatial stability is required. This paper discusses the design of a $200 \mathrm{~W}$ pre-stabilized laser (PSL) system and the underlying concepts. The PSL requirements for advanced gravitational wave detectors as well as for the laser system are described. The laser stabilization scheme proposed for the Advanced LIGO gravitational wave detector and the so-called diagnostic breadboard will serve as examples to explain the general laser stabilization concepts and the achieved performance and its limitations.

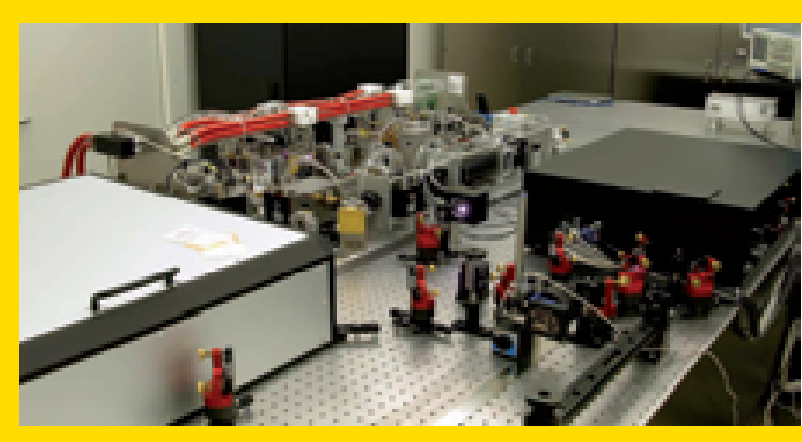

A $200 \mathrm{~W}$ high-power laser system developed for advanced gravitational wave detectors. The box on the left contains a $35 \mathrm{~W}$ front-end laser which is used to injection-lock the high-power stage (center of the photograph). The black box contains diagnostic tools to perform a comprehensive laser characterization.

\title{
Stabilized lasers for advanced gravitational wave detectors
}

\author{
Benno Willke* \\ Max-Planck-Institut für Gravitationsphysik (Albert-Einstein-Institut) und Leibniz Universität Hannover, Callinstr. 38, \\ 30167 Hannover, Germany
}

Received: 3 July 2009, Revised: 31 October 2009, Accepted: 3 November 2009

Published online: 20 January 2010

Key words: High-power single-mode solid-state lasers, laser stabilization, gravitational wave detectors.

PACS: 42.62.-b, 42.60.Pk, 04.80.Nn, 95.55.Ym

\section{Introduction}

The research related to gravitational wave detection is a fascinating, fast-growing field in physics. Due to the strong technological advances in precision interferometry the first direct detection is within reach and an increasing number of scientists work on source modeling, on the development of data analysis methods and on experimental techniques to improve the sensitivity of current detectors.

Currently a search for gravitational waves is being performed by an international network of large-scale laserinterferometric gravitational wave detectors (GEO600 [1], LIGO [2], TAMA300 [3] and Virgo [4]).

Although the scientific community is still awaiting the first detection, upper limits on the strength of gravitational waves emitted from several sources have been established [5]. One of the most interesting results so far is that the known pulsar in the Crab Nebula emits less than $4 \%$ of its overall energy loss in gravitational waves. This corresponds to a mass asymmetry of that pulsar of less than $10^{-4}$ [6]. A regularly updated list of observational results can be found on the publications website of the LIGO Scientific Collaboration [7].

While the data from the first long science runs are still being analyzed, upgrades to the long-baseline detectors are planned to improve their sensitivities and scientific reach for astrophysical sources. The Advanced LIGO Project [8] as well as the Advanced Virgo Project [9] aim for sensitivity improvements of a factor of 10 , which corresponds to an increase in the number of detectable sources by approximately 1000 . The installation phase for the advanced detectors will start in 2011 and several years of commissioning will be required to reach the design sensitivity. 
Gravitational waves will give astrophysicists a new tool for studying the universe, beyond the different forms of electromagnetic radiation that have been used to date. New insight into the dynamics of supernova explosions and into the inspiral and merger phase of massive binary objects like neutron stars or black holes can be gained from gravitational wave waveforms and from their combination with the results from numerical simulations. Detection of neutron star normal modes could bring us information on their equation of state, and the detection of a stochastic gravitational wave background would allow us to look further back in time than is possible with electromagnetic waves.

Although astrophysics was and is the main driving force for the development and improvement of gravitational wave detectors (GWDs), detector science has become a fascinating field in optics and precision metrology. Technology improvements in the field of low-loss dielectric mirror coatings (e.g. [10-12]), seismic isolation (e.g. [13-15]), lownoise high-power lasers (e.g. [16]) and mechanical oscillators with high quality factors (e.g. $[17,18]$ ) were strongly influenced by research devoted to meeting the demanding requirements of GWDs. Fundamental studies on thermal noise in mechanical systems (e.g. [19-22]) led to a much deeper understanding of the underlying physics. Also, the areas of quantum non-demolition (QND) measurements (e.g. [23, 24]) and squeezed-light sources (e.g. [25]) were strongly influenced by the possible application of these techniques in GWDs. Furthermore the sensitivity of GWDs and prototype interferometers is approaching a level that will allow us to study mirrors as quantum objects (e.g. [26-28]).

For many years large, ton-scale cryogenic resonant mass detectors [29] dominated the field until laser interferometers surpassed the sensitivity of those so-called bar detectors. The fundamental noise source of laser interferometric GWDs directly related to the laser light is the shot noise in the interferometer readout. As the signal-to-noise ratio of the readout noise can be improved by higher light power in the interferometer, GWDs need high-power lasers as light sources. First-generation GWDs use lasers with light power of approximately $10 \mathrm{~W}[30,31]$ and secondgeneration GWDs require power levels of the order of $200 \mathrm{~W}$. In general, increasing the light power in the interferometer improves the sensitivity until the noise introduced by fluctuating radiation pressure forces on the mirrors reaches the same level as the readout noise. At this power level the so-called standard quantum limit (SQL) is reached. The response of the suspended mirrors to fluctuating radiation pressure forces depends on the mirror mass and on the Fourier frequency. Hence the value of the SQL as well as the power level at which the SQL is reached depend on these two parameters. Once the mirror masses are increased to the maximal feasible level, only QND techniques or the use of non-classical light sources can improve the sensitivity further. In addition to these quantum fluctuations, the technical power noise of the laser can couple into the main interferometer output (e.g. [32,33]). Frequency noise and spatial fluctuations of the laser beam can also limit the sensitivity of GWDs (e.g. [33,34]). Hence sophis- ticated techniques are required to stabilize the lasers by means of feedback control loops or passive filtering.

This paper is devoted to the light sources of GWDs and their stabilization. After a short introduction to the measurement principle of GWDs and a description of the requirements on their laser sources (Sect. 2), different options for high-power generation are reviewed (Sect. 3) with special emphasis on the scheme chosen for the Advanced LIGO 200 W laser system. Sect. 4 describes an integrated laser characterization tool and explains the stabilization scheme for the Advanced LIGO pre-stabilized laser (PSL). Although this scheme has features specific to Advanced LIGO, it can serve as an example for more general stabilization concepts. Almost all techniques are also applicable to fiber-based laser systems like the one currently under consideration for the Advanced Virgo GWD [9] or in many other fields that require highly stable laser systems. The paper ends with a summary and outlook of the future of lasers for GWDs (Sect. 5).

\section{Gravitational wave detectors and their light sources}

To fully understand gravitational waves one needs to study Einstein's theory of general relativity. Gravitational waves manifest themselves as ripples in the fabric of space-time. They are transversal quadrupole waves and can have two polarizations. Fig. 1 shows a schematic of the effect of a gravitational wave generated by a rotating binary system of two stars. The gravitational wave travels with the speed of light and changes the space-time fabric at a rate corresponding to twice the rotation period of the binary system.

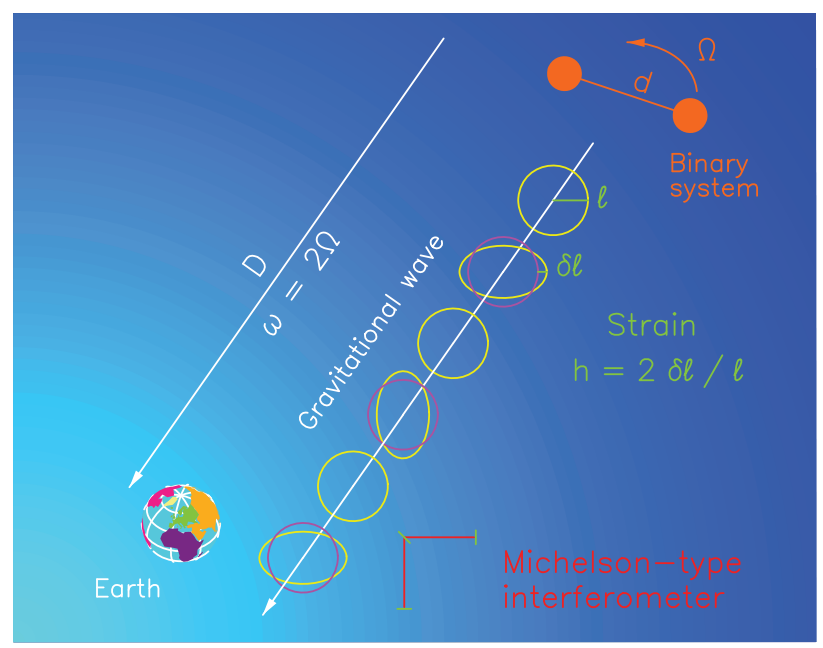

Figure 1 (online color at: www.lpr-journal.org) A gravitational wave generated by a rotating binary system of two stars causes space-time, and with it a circular arrangement of masses, to be deformed with a rate twice that of the rotation frequency of the stars. The strength of the gravitational wave can be described by the strain $h=2 \times \delta l / l$ 


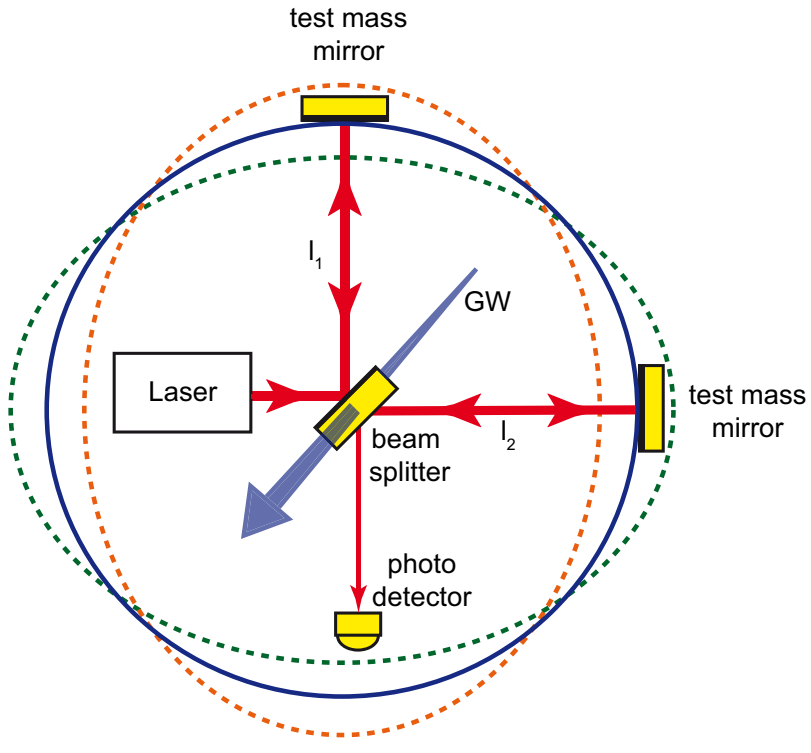

Figure 2 (online color at: www.lpr-journal.org) A gravitation wave (GW) with period $T$ changes space-time such that a circular arrangement of test masses (blue) changes to an ellipse (orange) at $T / 4$, back to the circle at $T / 2$, to the green ellipse at $3 T / 4$ and back to the circle at the end of a period. A Michelson interferometer with its beam splitter in the center of the circle is a perfectly matched instrument to measure the effect of a gravitational wave passing the Earth.

For an explanation of the measurement principle of GWDs let us assume we have a set of free masses arranged on a circle (see Fig. 2). When a gravitational wave passes this arrangement the space-time fabric changes in such a way as if the circle were transformed into an ellipse. A quarter of a period later the arrangement will look circular again and will then change into an ellipse now having its major axis perpendicular to the direction it had half a period earlier. The strength of a gravitational wave is given by the strain $h$ and can be quantified by the relation $h=2 \times \delta l / l$, where $\delta l$ is the difference between the radius of the circle and the long or short axis of the ellipse and $l$ is the radius of the circle. The expected strain $h$ of gravitational waves on Earth is very small with $h \leq 10^{-21}$, which is why they have not yet been detected.

A Michelson interferometer with its beam splitter in the center of the circle and the two arms aligned along the principal axes of the ellipse (see Fig. 2) is an ideal instrument to measure the differential change in arm length caused by a gravitational wave. The variation of the difference between the length of the two interferometer arms $\Delta l=l_{2}-l_{1}$ would cause a change in the light power $\delta I$ at the interferometer output port. The optical response of an interferometer defined as $\delta I(\Delta l)$ follows a $\cos ^{2}(\Delta l)$ relation and is plotted in Fig. 3. The power change on the output photodiode caused by a gravitational wave depends on the operation point of the interferometer, i.e. on the value of $\Delta l$ in the absence of gravitational waves. In Fig. 3 the response to a sinusoidal displacement is depicted for the so-called mid-fringe and dark-fringe operation point. Although the mid-fringe point gives the largest signal for a given change in arm length, all GWDs operate near the dark-fringe point. This is for several reasons, the most important of which is the strong coupling of laser power fluctuations into the interferometer output at the mid-fringe.

The leading term in the response at the dark port is quadratic, such that a harmonic displacement with a frequency $\Omega$ is converted into a photodiode signal at $2 \Omega$. At this operation point the directional information is lost which means that one cannot tell from the photodiode signal if the differential displacement became larger or smaller. This fact is in particular problematic for interferometer length control. In addition, at this operation point the tiny photocurrent produced by a potential gravitational wave would be much smaller than unavoidable technical noises. For-

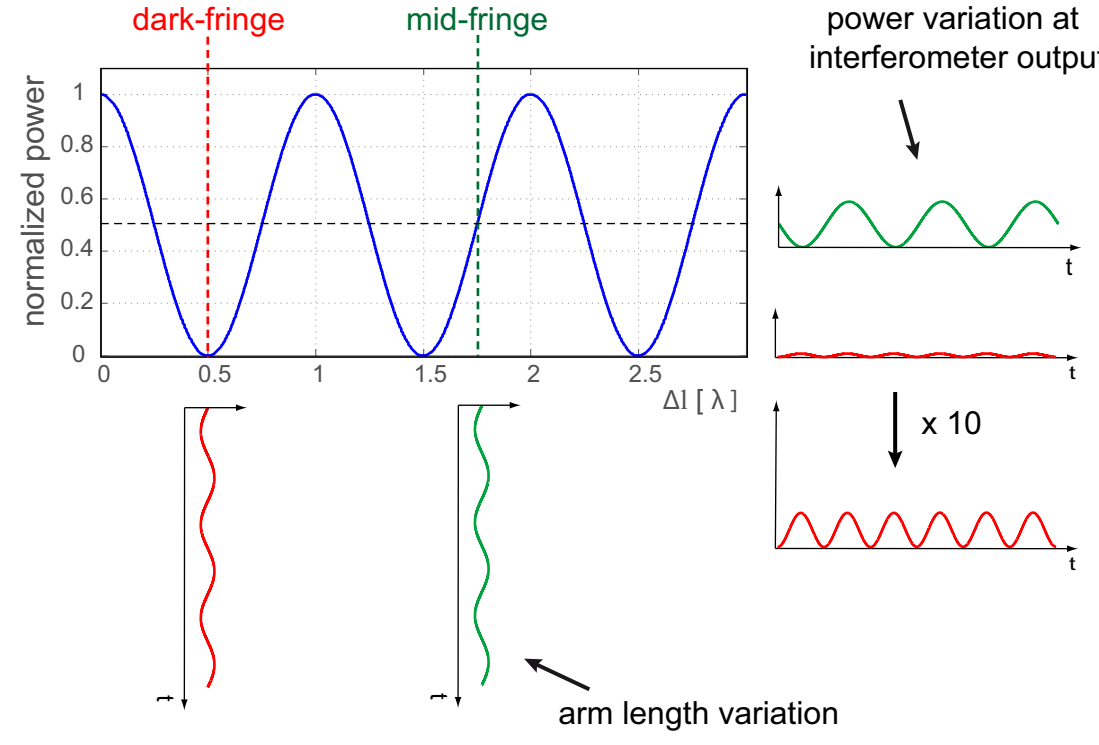

Figure 3 (online color at: www.lprjournal.org) Response of an interferometer to a differential displacement of its end mirrors or to a gravitational wave. Time series are plotted to visualize the dynamic response of an interferometer to a harmonic path-length variation at the dark-fringe and mid-fringe operation points. 
tunately both effects can be compensated using a heterodyne detection technique. A full description of the sensing scheme of GWDs is beyond the scope of this paper, but can be found in a book by Saulson [35]. The actual optical design of GWDs is much more complex than a simple Michelson layout. The fundamental concepts of different optical layouts (Fabry-Perot arm cavities, delay lines, power and signal recycling techniques) are also described in Saulson's book. A detailed description of the different GWDs is given in the status report of various projects [1-4] and references therein. Although the design of these GWDs is quite different, they all use solid-state lasers as light sources, all with similar noise requirements. The design and stabilization of such lasers will be the topic of the remaining part of this paper.

\section{High-power lasers for gravitational wave detectors}

The main challenges in the development of laser systems for use in GWDs arise from the fact that the high-power radiation has to have a diffraction-limited spatial profile and must be linearly polarized. Furthermore, the laser has to operate in a single-frequency mode with low fluctuations in power, frequency and beam pointing. The laser design has to incorporate wide-range, high-bandwidth actuators for stabilization purposes and it needs to ensure a high reliability, a soft failure mode and should be easy to maintain. To achieve these goals, several design choices have to be made and special techniques need to be applied.

The tasks of generating high laser power and to fulfill the single-frequency requirement are usually split by the use of a master oscillator power amplifier (MOPA) design or by injection locking a high-power oscillator to a single-frequency master laser. A Nd:YAG non-planar ring oscillator (NPRO) is commonly used as the master laser (see Fig. 4).

This laser type was invented by Byer and Kane in 1985 [36] and NPROs are commercially available with output powers of up to $2 \mathrm{~W}$ [37]. The non-planar resonator design in combination with an internal Faraday rotation,

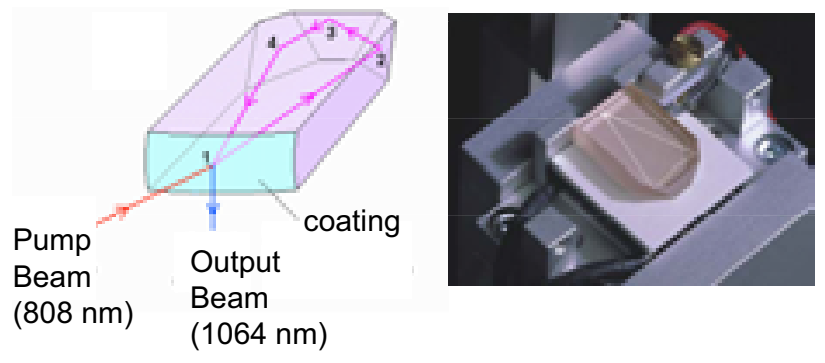

Figure 4 (online color at: www.lpr-journal.org) Schematic and image of a NPRO which is used as a highly stable master laser in all high-power laser systems for GWDs. achieved with a permanent magnet placed close to the crystal, provides all features required to form a unidirectional ring laser. Both the Faraday effect and the non-planar design cause a rotation of the polarization state of the circulating light field. The two effects, however, add for one traveling direction of the beam and subtract for the opposite one. This fact together with the polarization-dependent reflection coefficient of the front facet cause different round trip losses for the two directions. Since a homogeneous line broadening mechanism is dominant the light fields traveling in opposite directions can compete for the gain and the system responds with single directional operation. For large crystal temperature ranges the laser operates in a single-frequency mode and the monolithic design ensures small mechanical fluctuations in the cavity length. A small change in the crystal temperature caused by fluctuations in the power of the pump light was found to be the dominant source for the frequency noise of the NPRO [38]. This temperature change couples via the temperature dependence of the refractive index and, to a smaller extent, through the thermal expansion of the crystal, to the optical resonator length and by that to the laser frequency. A piezoelectric element mounted on top of the laser crystal can introduce a stress-induced refractive index change in the laser resonator and can hence be used as a tuning element for the laser frequency. For slow, widerange actuation of the laser frequency the well-controlled crystal temperature can be changed.

The relative power noise (RPN) of commercially available NPROs in the gravitational wave band $(10 \mathrm{~Hz}$ to $10 \mathrm{kHz}$ ) is RPN $\leq 1 \times 10^{-6} \mathrm{~Hz}^{-1 / 2}$, and each NPRO comes with an internal noise eater that suppresses the relaxation oscillation peak at around $1 \mathrm{MHz}$ and reduces the lowfrequency RPN by a factor of approximately five. Fig. 5 shows the noise spectral density of relative power noise of eight different NPRO units with the noise eater turned on, and an average taken over all units with noise eater off. Formally the noise spectral density is the Fourier transform of the autocorrelation function. For a stationary noise source the spectral density allows a much deeper insight into the characteristics of the noise than the time series. Spectral features in the spectral density point towards resonances in the noise coupling path and the overall curve reveals information on the spectral shape of either the noise source itself or the transfer function via which it couples into the signal under investigation.

In addition, NPROs have a good spatial beam profile with in most cases less than 3\% of the power in higher-order modes. They are slightly elliptically polarized and their polarization extinction ratio can, by means of retarding plates, be improved from $1 / 6$ to $1 / 100$. A detailed characterization of NPROs can be found in [39], which provides information on the unit-by-unit fluctuations of eight NPROs as well as day-by-day fluctuations of one unit over 3.5 months. To gain the day-by-day fluctuation results, one NPRO was automatically characterized each night. Frequency noise, power noise, pointing noise and the higher-order mode content were measured with a diagnostic breadboard (see Sect. 4). 


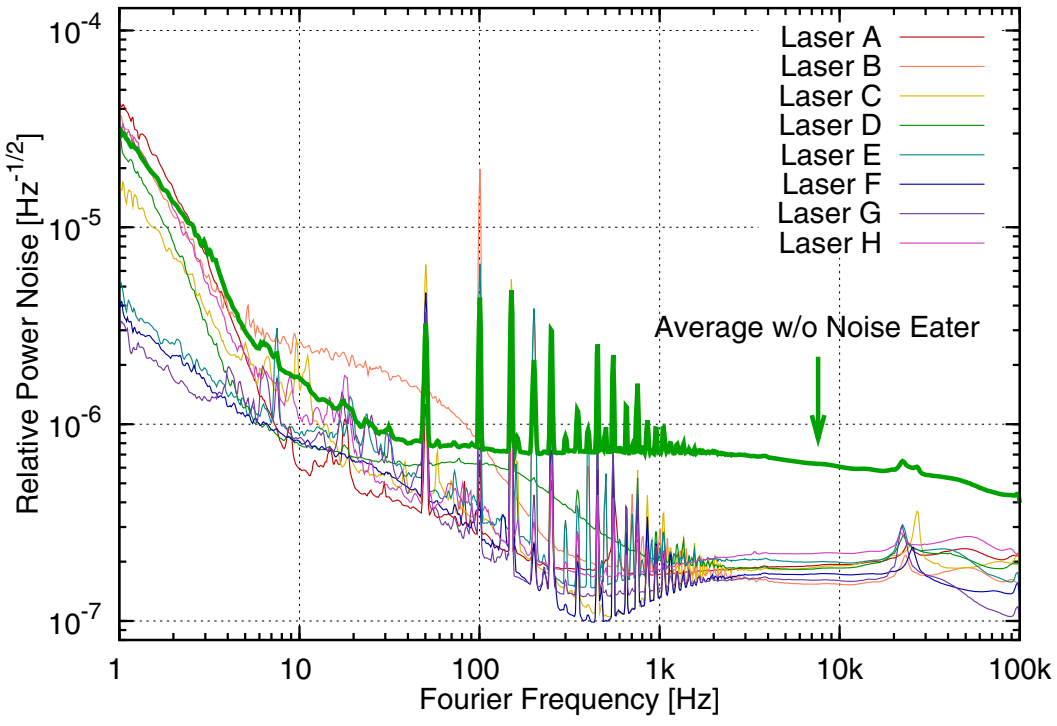

Figure 5 (online color at: www.lprjournal.org) Relative power noise of eight different $2 \mathrm{~W}$ NPRO lasers with operating noise eater and an average over all systems with the noise eater turned off. The noise eater is an internal power stabilization loop optimized to suppress the relaxation oscillation at frequencies of approximately $1 \mathrm{MHz}$.
Noise spectra and histograms of several lasers show that the NPROs are highly stable laser sources with only small variations between different samples. The performance described above makes an NPRO well suited as a master laser in a MOPA or injection-locked configuration.

One of the main objectives in high-power generation is to handle the thermal load in the laser crystals. Pump light is absorbed in the crystals and, due to the quantum defect, non-radiative decay and absorption processes starting from the upper laser state, a fraction of this power is converted into heat which has to be removed via a cooling interface. This effect unavoidably generates a temperature gradient and the resulting stress sets an upper limit to the acceptable pump power, as the stress needs to stay below the stress-fracture limit of the crystal material. The temperature gradient, combined with the temperature dependence of the refractive index, the thermal expansion of the crystal material and the photoelastic effect in the material, generates a thermal lens [40] that has to be taken into account in the laser design. Aside from its focusing power this lens normally shows large aberrations, and several concepts can be found in the literature to reduce the effect of these aberrations. One solution is to choose a zigzag path of the laser mode in the direction of the thermal gradient and hence to average over the thermal effects $[36,41,42]$. A second solution is to align the laser mode parallel to the thermal gradient and choose the crystal aspect ratio such that the mode radius is large compared with the crystal thickness. This concept is applied in thin-disc lasers [43]. In fiber lasers (e.g. [44]), the thermal problem is reduced by extending the length of the active medium and by confining the laser mode in the fiber. The concept chosen for the Advanced LIGO laser is a rod design which makes use of the aberrated thermal lens to improve the spatial beam profile, as will be described later.

The rod geometry has several advantages compared with the slab design. As the rod geometry matches the circular beam shape of the Gaussian laser mode, very good spatial overlap between the gain medium and the laser mode can be achieved, which in turn leads to a high efficiency of the laser. The good overlap furthermore reduces the regions with unsaturated gain that could support parasitic modes. Such modes can cause a degradation of the spatial beam quality in slab lasers, and a careful choice of the crystal angles and of the surface roughness of the slab is essential to achieve high output powers [45]. Closed paths for parasitic modes in rods are very unlikely as only the end faces have planar surfaces that could support such modes.

One key problem of rod crystal geometries in linearly polarized lasers is the depolarization caused by stressinduced birefringence $[46,47]$. The temperature gradient causes stress in the radial direction which leads to the fact that the principal axes of the birefringence are the radial and tangential directions (see Fig. 6). Hence a linearly polarized laser mode senses different refractive indices at different locations: p-polarized light for example senses $n_{\text {radial }}$ at the left and right side of the crystal whereas at the top and bottom it sees $n_{\text {tangential }}$. In addition $n_{\text {radial }}$ and $n_{\text {tangential }}$ depend in a different way on the temperature profile such that the polarized beam sees two thermal lenses with different focal length. This effect is called bi-focusing. At other locations, for example at the third position marked in Fig. 6, the laser beam senses a combination of $n_{\text {radial }}$ and $n_{\text {tangential }}$ such as if the rod was a retardation plate. Hence, if the rod is put between crossed polarizers a cloverleaf-shaped intensity profile as illustrated in Fig. 6 will be transmitted. The depolarization as well as the bi-focusing can limit the achievable output power of linearly polarized lasers.

Both effects, depolarization and bi-focusing, can be reduced to a large extent by choosing a naturally birefringent laser material such as neodymium-doped yttrium vanadate $\left(\mathrm{Nd}: \mathrm{YVO}_{4}\right)$, as was done in the case of the intermediate power stage of the Advanced LIGO laser [48,49]. In this laser system the NPRO light passes four $\mathrm{Nd}: \mathrm{YVO}_{4}$ crystals in an amplifier configuration (see Fig. 7). 


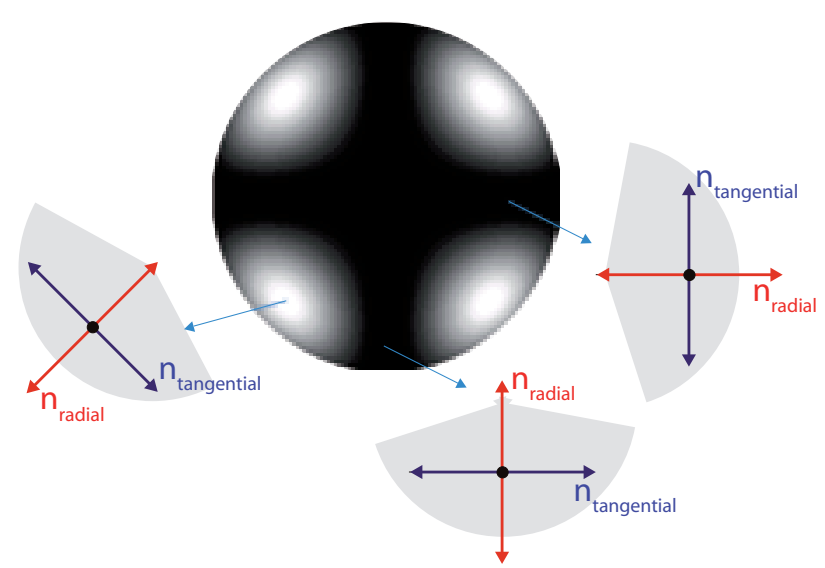

Figure 6 (online color at: www.lpr-journal.org) Explanation of the depolarization effect caused by radial stress in laser rods. The blue arrows show the polarization direction that senses the refractive index in the tangential direction and the red arrows correspond to the radial direction. The clover leaf intensity profile forms when a depolarized laser beam passes a polarizer.

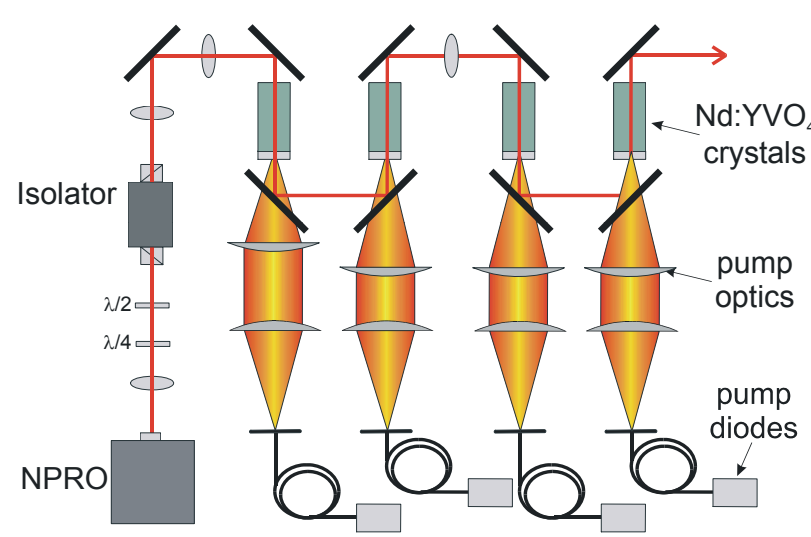

Figure 7 (online color at: www.lpr-journal.org) Schematic layout of a $35 \mathrm{~W}$ front-end laser system. An NPRO is amplified by four $\mathrm{Nd}: \mathrm{YVO}_{4}$ crystals each pumped with a fiber-coupled laser diode.

Each crystal is pumped by a $400 \mu \mathrm{m}$ diameter, NA:0.22 fiber-coupled laser diode with a nominal power of $45 \mathrm{~W}$. To increase the lifetime of these diodes the pump power is set to $33 \mathrm{~W}$ during normal operation. The laser crystals with a $3 \mathrm{~mm} \times 3 \mathrm{~mm}$ cross section consist of a $2 \mathrm{~mm}$ undoped and an $8 \mathrm{~mm}$ long doped $\mathrm{Nd}: \mathrm{YVO}_{4}$ region (0.3 at.\%). The un-doped region is bonded to the crystal to reduce thermal stress at the surface where the pump beam enters. To separate the pump light from the laser light, dichroic $45^{\circ}$ mirrors with an anti-reflection coating for the pumplight wavelength and a high-reflection coating for the laser wavelength are implemented. Efficient cooling of the laser crystals is realized by wrapping them into a $500 \mu \mathrm{m}$ thick indium foil and by mounting this assembly in water-cooled copper blocks. After passing the four laser heads the NPRO

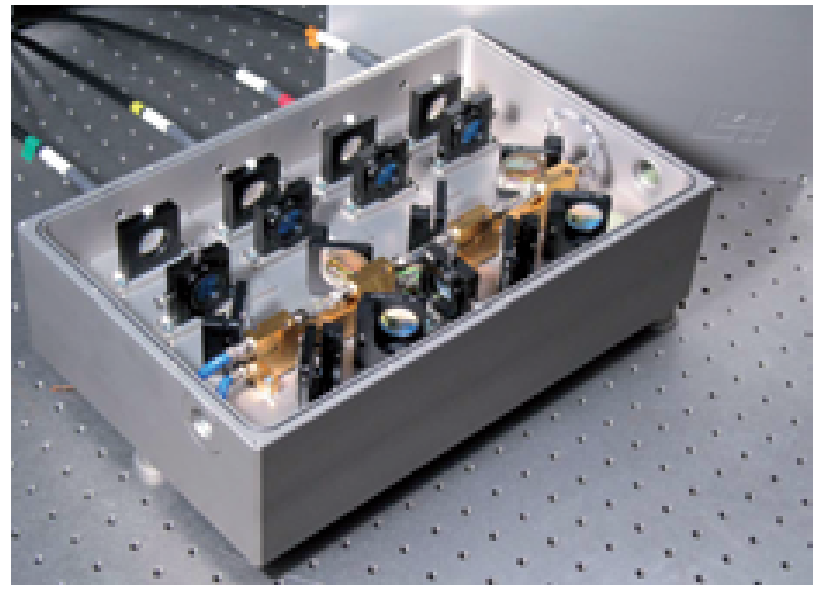

Figure 8 (online color at: www.lpr-journal.org) A $35 \mathrm{~W}$ $\mathrm{Nd}: \mathrm{YVO}_{4}$ amplifier stage. The laser crystals are mounted in watercooled copper blocks for conductive cooling.

$2 \mathrm{~W}$ beam is amplified to a level of $35 \mathrm{~W}$. In a power scaling experiment output power levels of up to $65 \mathrm{~W}$ for a seed power of $20 \mathrm{~W}$ were achieved [49]. An image of the amplifier stage is shown in Fig. 8.

Wavelength matching between the Nd:YAG and the $\mathrm{Nd}: \mathrm{YVO}_{4}$ system can be done by temperature tuning of the NPRO. As a result of the broad emission profile of $\mathrm{Nd}: \mathrm{YVO}_{4}$ of approximately $0.9 \mathrm{~nm}$ (FWHM), an efficient amplification was achieved over a large NPRO temperature range of $25-40{ }^{\circ} \mathrm{C}$ [49]. The pump spot diameter in each crystal was optimized to achieve a high extraction efficiency without degrading the spatial beam profile. The spatial beam quality of the NPRO $\left(97 \%\right.$ TEM $_{00}$ mode content) is only slightly degraded by the amplification process, and $95 \%$ of the power of the $35 \mathrm{~W}$ laser was measured to be in the $\mathrm{TEM}_{00}$ mode. This small degradation can be attributed to the third and fourth amplification stage, as after the first two stages a $\mathrm{TEM}_{00}$ mode content of $97 \%$ was measured. The combined system of the NPRO and the vanadate amplifier stages will be called the $35 \mathrm{~W}$ front end in the following. The RPN of the $35 \mathrm{~W}$ front end is identical to the NPRO RPN at frequencies below $50 \mathrm{~Hz}$ and exceeds it by a factor of up to five for frequencies in the audio band.

The high-power stage for the Advanced LIGO laser is an injection-locked oscillator with four laser heads. Each laser head consists of a water-cooled Nd:YAG rod and its pump unit (see Fig. 9). The rods are $3 \mathrm{~mm}$ in diameter and have a $40 \mathrm{~mm}$ long 0.1 at. \% doped region. Two un-doped, $7 \mathrm{~mm}$ long end-caps are bonded to their ends to reduce the heat load at the rod faces and to allow for a uniform cooling of the active region by supporting and sealing the rods at these un-doped ends. The pump units consist of seven fibercoupled $808 \mathrm{~nm}$ laser diodes, each with a nominal output power of $45 \mathrm{~W}$. As in the case of the front end, the laser diodes are operated at reduced output power to increase their lifetime. The light leaving the fiber bundle is coupled into a $2 \mathrm{~mm}$ diameter, $10 \mathrm{~mm}$ long fused silica rod to mix 

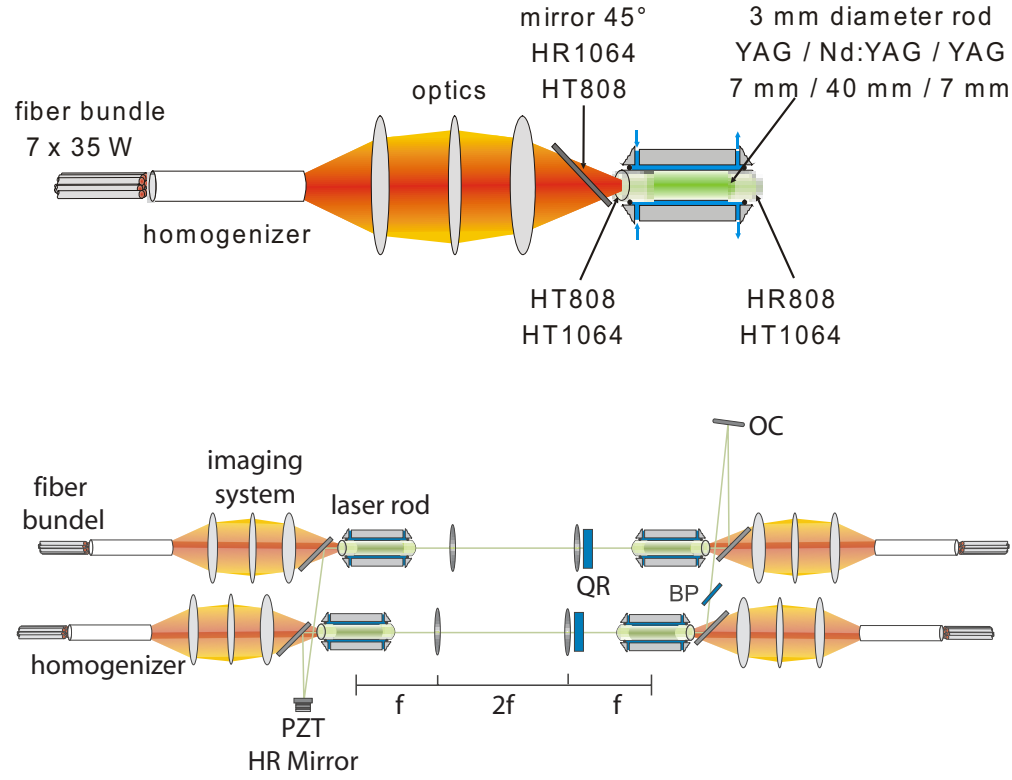

Figure 9 (online color at: www.lpr-journal.org) Each of the four laser heads of the $200 \mathrm{~W}$ laser consists of a homogenizer, pump-light imaging optics, a $45^{\circ}$ resonator mirror and water-cooled Nd:YAG rod The $808 \mathrm{~nm}$ pump light is brought to the laser head via seven pump fibers. An $808 \mathrm{~nm}$ high-reflective coating at one of the rod surfaces establishes a double-pass configuration and leads to high pumplight absorption.

Figure 10 (online color at: www.lpr-journal.org) Schematic of the $200 \mathrm{~W}$ high-power stage. The ring laser consists of four laser rods grouped in two pairs each with a depolarization compensation unit: two lenses and a quartz rotator (QR). Due to a Brewster plate (BP) the laser produces linear polarized light which is partly transmitted through the output coupler (OC). A mirror mounted on a piezoelectric actuator (PZT) can be used to injection lock this laser to the $35 \mathrm{~W}$ front end. the pump light of the different fibers and to produce a uniform intensity profile which avoids hot-spots in the crystal. An additional benefit of this homogenizer is the fact that the spatial pump-light distribution does not change when one laser diode fails. In that case the reduced power can be compensated by the remaining six diodes and the faulty diode can be replaced at a convenient time. A telescope focuses the pump light into the laser rod where the pump light is guided by total internal reflection, as the water-cooled rod surface acts as a waveguide. A highly reflective coating at $808 \mathrm{~nm}$ on the end-face of the rod leads to a double-pass configuration for the pump light. This has the advantage of a more uniform pump-light absorption along the crystal axis than a single-pass arrangement would provide [50].

Fig. 10 shows a schematic layout of the complete highpower stage. The bi-focusing and birefringence problem mentioned above can be almost completely suppressed using the following compensation scheme [51]: the four rods are grouped in two pairs, each with a lens combination that images one rod of a given pair into the second one. In addition, a quartz rotator changes the polarization state of the beam between the two rods of a pair by $90^{\circ}$. Hence the light has sensed an equal distance of $n_{\text {radial }}$ and $n_{\text {tangential }}$ by traveling through a rod-rotator-rod unit. This scheme works almost perfectly for a two-head pair as described in [50]. Equally good results are achieved in the four-head Advanced LIGO laser and almost no light in the characteristic depolarization pattern is reflected by the Brewster plate that serves as a polarizer in the resonator. An aperture in the resonator [52] or a sophisticated design of the stability ranges of the high-power resonator [48] ensures a fundamental-mode operation of the laser. The latter makes use of the aberrations in the thermal lens which are more relevant for higher-order spatial modes than for the fundamental mode. Hence higher-order modes sense a different effective focal length of the thermal lens. Based on this difference a specific pump power together with a specific resonator design can be chosen, such that the diffraction losses for higher-order spatial modes are higher than for the $\mathrm{TEM}_{00}$ mode, and that the laser thus operates in its fundamental mode.

An NPRO, a $35 \mathrm{~W}$ amplifier and the injection-locked high-power oscillator form the Advanced LIGO laser system, a photograph of which is shown in Fig. 11. A prototype version of this laser system delivers more than $200 \mathrm{~W}$ output power and an automatic lock-acquisition process takes typically less than $10 \mathrm{~ms}$. Once injection-locked, a control loop feeding back to a fast piezoelectric actuator and to a slow long-range actuator keeps the system in lock for several days until an external disturbance occurs. To investigate the long-term behavior of the system, frequent characterization measurements are performed with a diagnostic breadboard, which is described in Sect. 4.

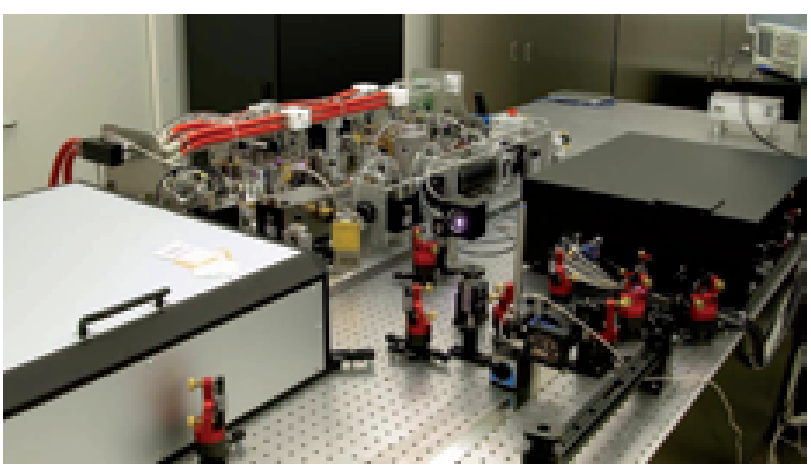

Figure 11 (online color at: www.lpr-journal.org) A 200 W highpower laser system. The box on the left contains a $35 \mathrm{~W}$ front end that is used to injection-lock the high-power stage (left side). The black box contains the diagnostic breadboard used to perform a comprehensive laser characterization. 


\section{Laser beam characterization and stabilization}

Lasers for use in GWDs have to fulfill exceedingly stringent requirements concerning their stability and reliability. The frequency stability in the interferometer needs to be at a level of several $\mu \mathrm{Hz} \mathrm{Hz}^{-1 / 2}$ which corresponds to a relative stability of $10^{-20} \mathrm{~Hz}^{-1 / 2}$, and RPN values as low as RPN $\leq 2 \times 10^{-9} \mathrm{~Hz}^{-1 / 2}$ have to be reached at a Fourier frequency of $10 \mathrm{~Hz}$. Such extreme stability levels can only be achieved by starting with a low-noise laser system that is then further stabilized by means of nested control loops with sophisticated sensing schemes. The laser stabilization scheme employed for the Advanced LIGO detector is one possible way to meet such demanding requirements. The key elements of this stabilization scheme will be described in the next section of this paper.

For the design of the laser stabilization as well as for the optimization process of the laser system itself, an accurate characterization of the laser performance is essential. Especially for complex systems such as the four-head highpower injection-locked laser described above, frequent characterization measurements have to be performed. Small changes in the resonator alignment can influence many beam parameters, as, for example, the higher-order mode content, the RPN or the beam pointing fluctuations. Whenever the alignment is changed during, for instance, a power optimization process, a full characterization of the laser is required to determine possible effects of the new alignment on the other beam parameters. Furthermore, a fast and accurate characterization ability is a prerequisite for drift investigations and for short GWD down-times during laser maintenance work. Hence we developed a fast, easy-to-use and automated characterization tool capable of measuring the beam parameters to the required precision.

\section{The diagnostic breadboard}

The diagnostic breadboard (DBB) is a tool for performing comprehensive laser characterization. The heart of the DBB (see Fig. 12) is a rigid-spacer ring resonator that serves as a modal and frequency reference. The design of this resonator is similar to the one described in [53]. The Pound-DreverHall method $[54,55]$ is used to obtain information on the difference $\Delta \nu$ between the frequency of the laser under test and the frequency of the appropriate fundamental mode resonance of the resonator. In this technique a laser beam with phase modulation sidebands is aligned to match the eigenmode of the optical resonator. ${ }^{1}$. Most of the carrier

\footnotetext{
${ }^{1}$ The sideband picture is used in this paper as it allows one to gain an intuitive understanding of the behavior of light fields with different frequencies under interference conditions and in situations that involve optical elements with a narrow line width and an associated strong dispersive behavior. The main fraction of the light field will be called the carrier and electric fields at slightly larger/lower frequencies the upper/lower sideband, respectively.
}

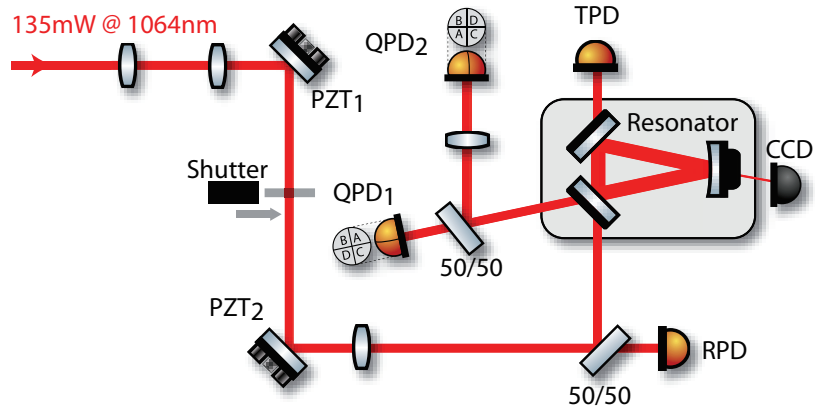

Figure 12 (online color at: www.lpr-journal.org) Schematic overview of the diagnostic breadboard. An optical resonator defines the spatial and frequency reference against which the laser under test is compared. The modal content is analyzed with a transmission photodiode (TPD) and a CCD camera. Quadrant photodiodes (QPD) measure the pointing fluctuations and piezoelectricactuated steering mirrors (PZT) are used as part of an automatic alignment system. The power fluctuations are measured with a photodiode (RPD).

light field is directly reflected at the in-coupling mirror of the resonator. A smaller fraction is transmitted through this mirror, travels around the cavity, is then reflected at the in-coupling mirror and can interfere with the light field that enters the cavity via the in-coupling mirror one round-trip time later. If the phases of the two fields match, e.g. if the round-trip length is an integer of the wavelength, the light is said to be on resonance. The light fields that successively enter the cavity interfere constructively and the field inside the cavity can, in a steady-state situation, build up to a much higher level than the field strength of the injected field. A fraction of this field now leaks out of the cavity via the slightly transmissive in-coupling mirror and can interfere with the directly reflected light mentioned earlier. The amplitude and phase of this combined carrier field depends on $\Delta \nu$ and on the impedance matching of the cavity. (For a comprehensive description of optical cavities see standard textbooks such as $[56,57]$.) A further interference between the reflected, non-resonant sidebands and the combined carrier leads to a power modulation of the light at the sideband frequency, which can be detected with a photodiode. The size and phase of the photocurrent now carries the information on $\Delta \nu$, and a subsequent electronic mixing process results in an electrical signal that is proportional to $\Delta \nu$ (at least as long as $\Delta \nu$ is smaller than the cavity's linewidth). A control loop feeding back to the resonator length via a piezoelectric actuator can be engaged to keep the cavity resonant with the laser beam, and the error and control signals of this loop provide the desired information on the frequency noise of the laser. The sensitivity of this frequency noise measurement, which is limited by the length noise of the resonator, is sufficient to measure the fluctuations of the non-stabilized Advanced LIGO laser. By putting this resonator into a sealed metal tank, its length fluctuations can be reduced to a level at which the associated fluctuations of its resonance frequency are, by about 
one order of magnitude, below the free-running noise of an NPRO. Evacuation of the tank does not improve this level further.

The beam reflected by the locked resonator is split into two paths and aligned onto two quadrant photodiodes. These diodes are used in a differential-wavefront sensing scheme [58] to gain information on the relative pointing of the laser beam in regard to the eigenmode of the resonator. Four degrees of freedom are relevant, which can be interpreted as a tilt and as a parallel shift of the laser beam in the horizontal and vertical direction. By means of four control loops including two piezoelectric-actuated steering mirrors, the laser beam is automatically aligned to be collinear with the resonator mode. The error and control signals of these loops are then combined to estimate the pointing fluctuations of the laser under test. These control loops furthermore ease the mode matching of the laser into the resonator as the beam is always kept perfectly aligned during the adjustment of the mode-matching lens positions. After the mode matching is optimized, the length and alignment loops are disabled and the length of the resonator is scanned over several free spectral ranges. The transmitted light power is monitored and a theoretical curve is automatically fitted to the measurement to calculate the strength of higher-order modes that are resonant at different lengths of the non-degenerated resonator. Fig. 13 shows two example measurements. In the upper curve a spatially pre-filtered NPRO beam is characterized to demonstrate the sensitivity limit of the DBB and its analysis software. The total higher-order mode content of the beam is $0.18 \%$ and the smallest higher-order modes found by the software correspond to a fractional power level of $10^{-5}$. In the lower curve the spatial profile of a $148 \mathrm{~W}$ photonic crystal fiber amplifier [59] is analyzed. CCD images of the strongest higher-order modes are shown as insets. Both graphs show only the measured transmitted power and the fundamental mode fit. All curves representing the higher-order mode fits are omitted for clarity. As discussed in [60], such mode scans are the only accurate way to measure the higher-order mode content of a laser beam, and this technique is superior to conventionally used $\mathrm{M}^{2}$ measurements or Gaussian beam fits to CCD camera images. Furthermore some special features of the resonator design allow one to obtain information on the beam ellipticity and astigmatism [60].

Almost half of the power injected into the DBB is aligned onto a high-power InGaAs photodiode (RPD in Fig. 12) which can be used to measure the power noise of the laser up to $70 \mathrm{MHz}$ with a shot noise limited sensitivity of $3 \times 10^{-9} \mathrm{~Hz}^{-1 / 2}$.

A computer-controlled measurement sequence can be initiated that performs a series of consecutive measurements of almost all beam parameters described above. Only the high-frequency power noise has to be measured separately with a spectrum analyzer. Noise spectral densities, drifts and error bars for the measured quantities can then be calculated. The DBB has proven to be a versatile instrument and will be an integral part of each Advanced LIGO PSL.
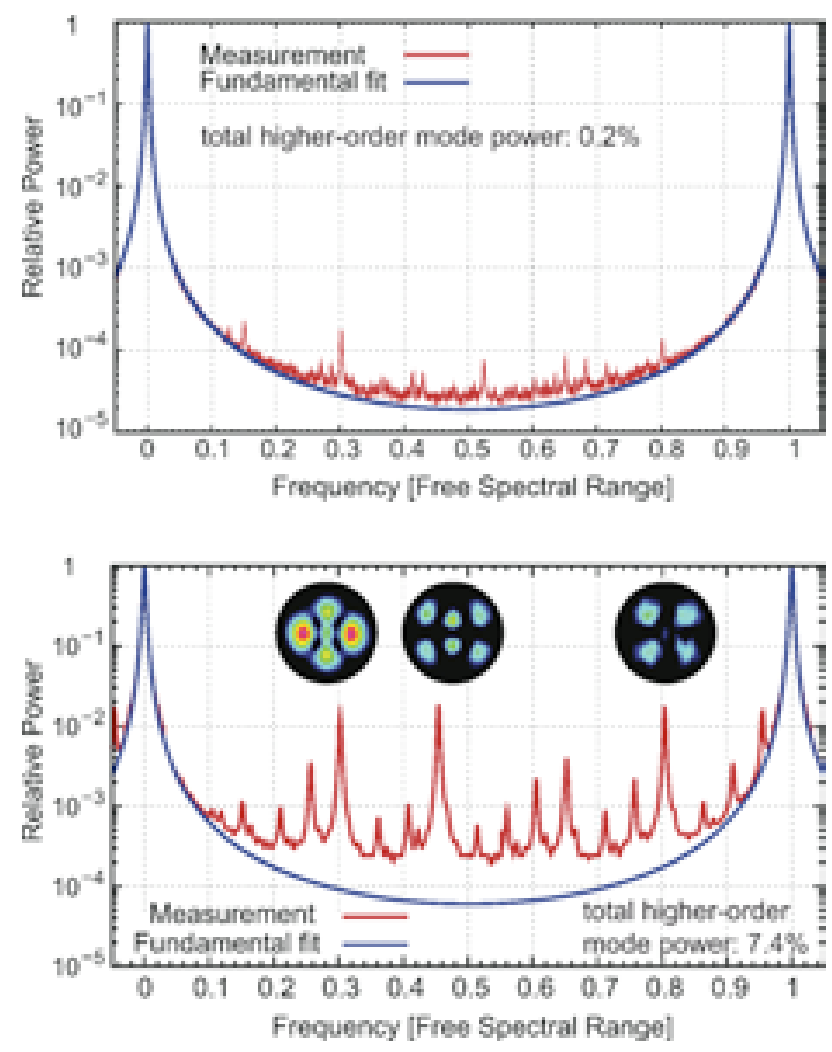

Figure 13 (online color at: www.lpr-journal.org) A modal analysis performed with the DBB. In the upper graph a spatially pre-filtered NPRO beam is characterized. Only $0.2 \%$ of its power is found in higher-order modes. The lower graph shows the modal content and of a $148 \mathrm{~W}$ photonic crystal fiber amplifier with images of the dominant higher-order modes.

It has been used to measure the fundamental-mode content of several NPROs (typically 97\%), several $35 \mathrm{~W}$ front-end lasers (typically 96\%), a $180 \mathrm{~W}$ high-power stage (around $90 \%$ ) and a $148 \mathrm{~W}$ photonic crystal fiber laser (92\%), and can be a useful supplement to many laser systems. More results of DBB measurements can be found in $[39,60]$.

\section{Spatial filtering and frequency stabilization}

A medium finesse rigid-spacer ring cavity, called the premode cleaner (PMC), is implemented in the Advanced LIGO PSL (see Fig. 14) to reduce the higher-order mode content of the laser beam. As the largest fraction of the spatial filtering of the laser is performed further downstream inside the GWD vacuum system by the suspended mode cleaners, only a small pre-filtering of the beam is required within the PSL. In the Advanced LIGO case the PMC has to filter higher-order modes of the laser beam to a level of $\leq 5 \%$ and needs to reduce the beam pointing of the laser by a factor of several hundreds. Both tasks can be achieved using a PMC with a finesse of $F \approx 100$. 


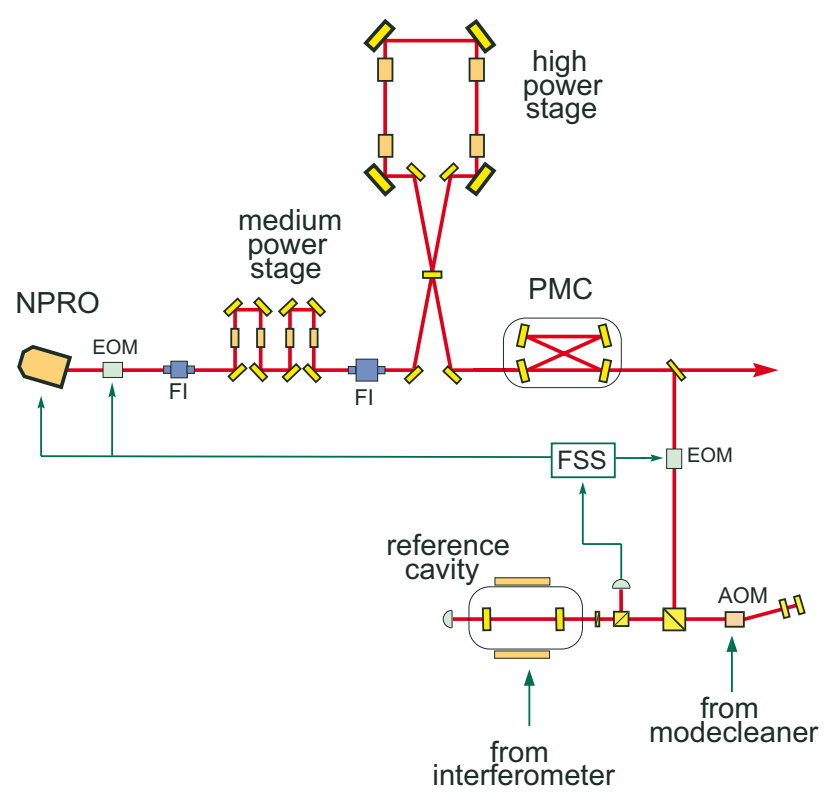

Figure 14 (online color at: www.lpr-journal.org) Layout of the Advanced LIGO PSL system including a schematic of the nested frequency control scheme. EOM: electro-optical modulator; AOM: acousto-optical modulator; FI: Faraday isolator; PMC: pre-modecleaner resonator; FSS: frequency stabilization electronics.

The PMC furthermore provides some filtering of RPN at the rf-frequencies at which additional phase modulation sidebands are imprinted on the laser beam downstream of the PSL. (These sidebands are used in Pound-Drever-Hall sensing schemes for the length and alignment control of the interferometer.) The power noise filtering of a Fabry-Perot cavity can be described by

$$
H_{\mathrm{T}}(f)=\frac{1}{\sqrt{1+\left(f / f_{\mathrm{c}}\right)^{2}}}
$$

where $H_{\mathrm{T}}(f)$ is the power fluctuation transfer function for light transmitted through the cavity at the Fourier frequency $f$ and $f_{\mathrm{c}}$ is the cavity half-width at half-maximum (HWHM). An intuitive understanding of this filter function can be gained in the sideband picture ${ }^{2}$. If the PMC is resonant for the carrier light, the transmission of sidebands at a HWHM frequency is reduced by a factor of $\sqrt{2}$. The power modulation of a beam is composed of the beat of the carrier with the modulation sidebands and therefore the power noise at a Fourier frequency of a HWHM is filtered by a factor of $\sqrt{2}$. In the Advanced LIGO case a HWHM for the PMC of approximately $580 \mathrm{kHz}$ was chosen to provide a power noise filtering of a factor of 15 at the modulation frequency of $9 \mathrm{MHz}$.

Lasers with small frequency fluctuations are required in several different fields of quantum optics and metrology such as in ultra-stable optical clock design, high-precision

\footnotetext{
${ }^{2}$ It should be noted that the sideband strength describes the amplitude of the electric field and not the light power.
}

spectroscopy and quantum state engineering (e.g. [61-64]). However, none of these experiments can reach the frequency stability of the GWD lasers in the $10 \mathrm{~Hz}-10 \mathrm{kHz}$ region. This is mainly attributable to the high stability and small linewidth of long-baseline high-finesse cavities of GWDs. These cavities provide seismically well-isolated frequency references for Fourier frequencies above the resonances of their mirror pendulum suspensions. At lower Fourier frequencies, however, most GWDs rely, as precision metrology experiments, on environmentally well-isolated rigid-spacer reference cavities (e.g. [65-67]). Interestingly the fundamental stability limit of such reference resonators was found to be related to coating thermal noise [68], which is also a limiting noise source in GWDs ${ }^{3}$. Hence scientists from the GWD as well as from the metrology community started to work in a combined effort with coating vendors to reduce the thermal noise in dielectric coatings while maintaining their high optical quality [69].

The goal for PSLs of GWDs is to achieve an intermediate frequency stability level and to provide high-bandwidth actuators for control loops that use the long GWD cavities as frequency references. In the Advanced LIGO case (see Fig. 14) the laser is pre-stabilized to a rigid-spacer highfinesse reference cavity that is suspended in a thermally controlled vacuum chamber on the laser table. For this purpose a small fraction of the power is picked off the main laser beam and is injected into the reference cavity after a set of phase modulation sideband are imprinted on the beam with an electro-optical modulator (EOM). The previously described Pound-Drever-Hall method is used to determine the deviation of the laser frequency from one of the resonant frequencies of the reference cavity, and this information is used in a control loop to minimize this deviation. The control signal is fed back to the piezoelectric frequency actuator of the NPRO and to a second EOM placed between the NPRO and the $35 \mathrm{~W}$ amplifier, which can be used as a fast phase-correcting actuator. A double-passed acoustooptical modulator (AOM) is placed between the pick-off point and the reference cavity such that it can change the frequency of the main laser beam with respect to the beam that is stabilized to the reference cavity. This AOM together with the temperature control of the reference cavity serve as actuators to stabilize the laser to the suspended mode cleaner and the interferometer.

\section{Power noise reduction}

Various mechanisms coupling laser power fluctuations into the gravitational wave channel exist in advanced GWDs. These coupling paths depend strongly on the interferometer design and can be divided into two classes. The first class couples directly via fluctuations in the light power detected

\footnotetext{
${ }^{3}$ Coating thermal noise is associated with mechanical motion of the dielectric coating stack and hence with a displacement of the effective point of reflection. This motion is activated by thermodynamic fluctuations in the solid.
} 
on the dark port photodiode. In a real interferometer there is always at least a small amount of light on this photodiode, for example due to intentional or accidental deviation from the dark-fringe operation point of the GWD. In this case a change of the light power injected into the GWD would cause a change in the power on the output photodiode and would be interpreted as a gravitational wave signal.

The second class couples via radiation pressure fluctuations into the position of the suspended interferometer mirrors. Although the beamsplitter ideally causes the power fluctuations on the laser beam to be split symmetrically into both interferometer arms, any asymmetry in, for example, the optical losses in the arms can break this balance. This would result in a difference in light power in the two arms and in the radiation pressure force on the suspended mirrors. Correspondingly a differential mirror displacement would occur which is then falsely interpreted as a gravitational wave. Even without any technical RPN on the light entering the interferometer, the quantum mechanical description of the beamsplitter results in uncorrelated shot noise in the arms which sets a lower limit on the radiation pressure noise.

Experimental investigations of existing GWDs [32] as well as simulations for advanced detectors [33] show complex features in the power noise coupling to the interferometer output. Coupling of power fluctuations into length and alignment control loops makes the situation even more complex such that only a detailed simulation of the interferometer behavior can reveal the frequency-dependent RPN requirement for advanced GWDs.

The most stringent requirement in the Advanced LIGO PSL was calculated to be RPN $\leq 2 \times 10^{-9} \mathrm{~Hz}^{-1 / 2}$ at $10 \mathrm{~Hz}$. As the main challenge of power stabilization experiments is the sensing of the power fluctuations, first stabilization experiments were performed with low-power lasers. Several such experiments [70-72] were limited by unexplained excess noise. Only recently could the required stability level be demonstrated [73]. In an NPRO power stabilization experiment extreme care was taken in the design of the electronics and in the reduction of scattered light in the optical setup. The laser beam was spatially filtered before it was detected with a multi-photodiode detector and the experiment was performed in a dust-free environment. With this setup we were able to achieve a RPN level of better than $\mathrm{RPN} \leq 1.7 \times 10^{-9} \mathrm{~Hz}^{-1 / 2}$ for frequencies between $10 \mathrm{~Hz}$ and $1 \mathrm{kHz}$.

Fig. 15a shows the basic principle of a power stabilization setup. A fraction $P_{\text {in }}$ of the main laser beam $P_{\text {laser }}$ is sent to a photodetector $\mathrm{PD}_{1}$. This photodetector measures the fluctuations and provides the error signal for a control loop filter, which sends the control signal to the laser to compensate for the fluctuations measured on $\mathrm{PD}_{1}$. In most experimental situations the beam transmitted by the beamsplitter is sent to the main experiment and it is often assumed that the fluctuations measured on $\mathrm{PD}_{1}$ are identical to the fluctuations on the beam sent to the main experiment. This assumption fails for fundamental reasons a)

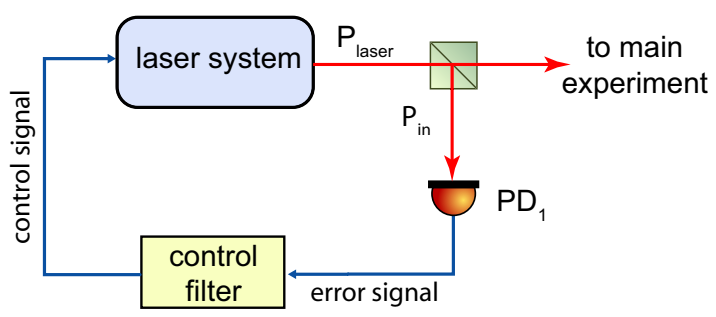

b)

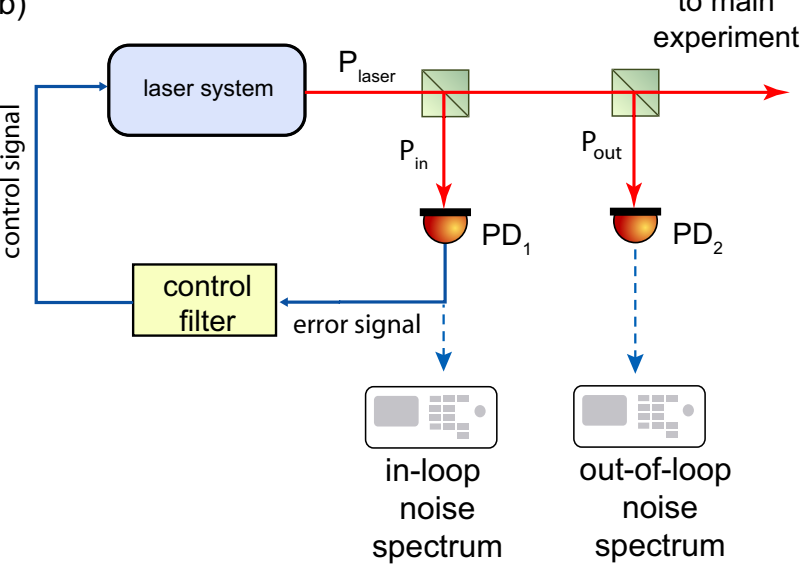

Figure 15 (online color at: www.lpr-journal.org) a) Principle of a power stabilization experiment. b) The same power stabilization setup with an out-of-loop detection which typically shows that larger fluctuations remain on the light beam than the in-loop measurement would suggest.

if one enters the shot noise limited regime and fails for technical reasons if noise sources add independent fluctuations on the sensing beam reflected by the beamsplitter and/or on the transmitted beam. Hence it is important to perform a so-called out-of-loop measurement with an independent photodetector $\mathrm{PD}_{2}$ as indicated in Fig. 15b.

One example of a technical noise source would be electronic noise of the photodetectors. The electronic noise $\tilde{N}_{1}^{\text {e }}$ of $\mathrm{PD}_{1}$ adds to the error signal, and the control loop will impress equivalent fluctuations with opposite sign onto the laser beam. In a steady-state situation the impressed fluctuations cancel the original noise and its appearance in the error signal will be reduced by the loop gain $G$ to $\tilde{N}_{1}=\tilde{N}_{1}^{\mathrm{e}} / G$. The electronic noise of $\mathrm{PD}_{1}$ will, however, now show up as power noise in $P_{\text {laser }}$ and can be sensed by $\mathrm{PD}_{2}$. (This means that the noise on the beam sent to the main experiment in Fig. 15a is not necessarily equal to the RPN measured on $\mathrm{PD}_{1}$.) $\mathrm{PD}_{2}$ has its own electronic noise $\tilde{N}_{2}^{\mathrm{e}}$ which we assume to be independent of the noise of $\mathrm{PD}_{1}$ and hence both will add in an incoherent way in the output of $\mathrm{PD}_{2}$ to $\tilde{N}_{2}=\sqrt{\left(\tilde{N}_{1}^{\mathrm{e}}\right)^{2}+\left(\tilde{N}_{2}^{\mathrm{e}}\right)^{2}}$, and if we assume that $\tilde{N}_{1}^{\mathrm{e}}=\tilde{N}_{2}^{\mathrm{e}}=\tilde{N}^{\mathrm{e}}$ we get $\tilde{N}_{2}=\sqrt{2} \times \tilde{N}^{\mathrm{e}}$. 


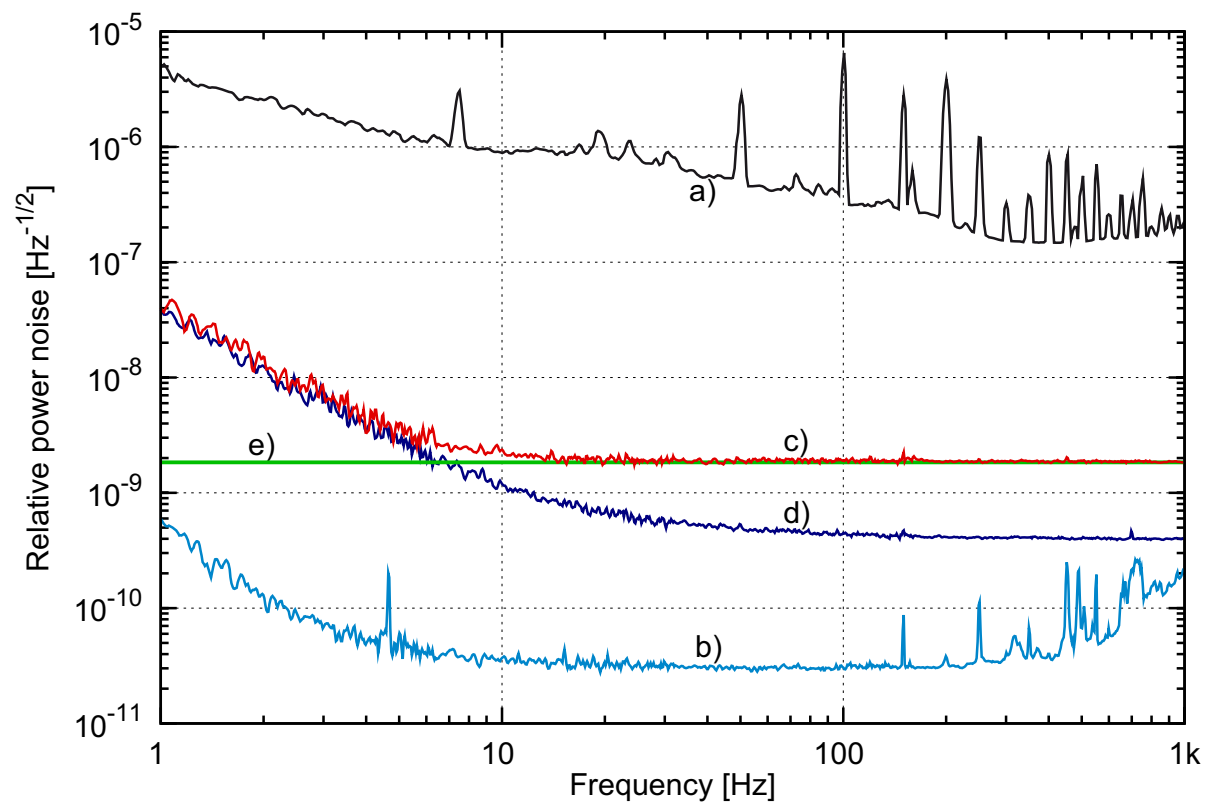

Figure 16 (online color at: www.lpr-journal.org) Linear spectral density of relative power noise measured in an NPRO power stabilization experiment. (a) Unstabilized noise, (b) in-loop noise measurement, (c) out-of-loop result, (d) electronic noise and (e) estimated shot noise.

Fig. 16 shows the result of the power stabilization experiment mentioned above [73]. Curve a shows the so-called free running noise of an NPRO laser without stabilization. Curve $\mathrm{b}$ shows the noise spectral density measured with the in-loop detector when the control loop is closed. As mentioned above it represents the free-running noise divided by the loop gain. Curve $\mathrm{c}$ is the out-of-loop noise measured with photodetector $\mathrm{PD}_{2}$. Curve $\mathrm{d}$ shows the projected electronic noise (incoherent sum of $\tilde{N}_{1}^{\mathrm{e}}$ and $\tilde{N}_{2}^{\mathrm{e}}$ ), and the expected shot noise is indicated by curve $\mathrm{e}$.

The measurement shows that the out-of-loop noise is very close to the projected electronic noise at frequencies below $5 \mathrm{~Hz}$. At higher frequencies the shot noise of the two photodetectors clearly explains the level of the achieved power stability. The shot noise was calculated from the measured DC photocurrent of $200 \mathrm{~mA}\left(\mathrm{PD}_{1}\right)$ and $189 \mathrm{~mA}$ $\left(\mathrm{PD}_{2}\right)$. To handle such high photocurrents, which correspond to light power levels of almost $250 \mathrm{~mW}$, we used arrays of four photodiodes for each photodetector $\left(\mathrm{PD}_{1}\right.$ and $\mathrm{PD}_{2}$ ).
A number of effects had to be considered to avoid additional noise in the light sensing. Some examples are pointing of the beam on the photodiode in combination with photodiode non-uniformities, polarization fluctuations in combination with a polarization-dependent splitting ratio of the pick-off mirror, scattered light with a fluctuating phase that beats with the main beam under investigation and electronic noise. All these effects were suppressed to a sufficiently low level in our experiment.

The results discussed above were achieved utilizing an NPRO as the laser source and an electro-optical amplitude modulator as the actuator in the power stabilization loop. Due to the greater complexity and the higher power level, a more complicated control loop design is required for a $200 \mathrm{~W}$ class GWD laser. Again the Advanced LIGO prestabilization concept will serve as an example (see Fig. 17).

Starting from an expected free running RPN of approximately $10^{-3} \mathrm{~Hz}^{-1 / 2}$ at $10 \mathrm{~Hz}$ a nested control loop with more than $110 \mathrm{~dB}$ loop gain has to be designed. The sensing point is placed at a leakage port of the PMC (see Fig. 17)

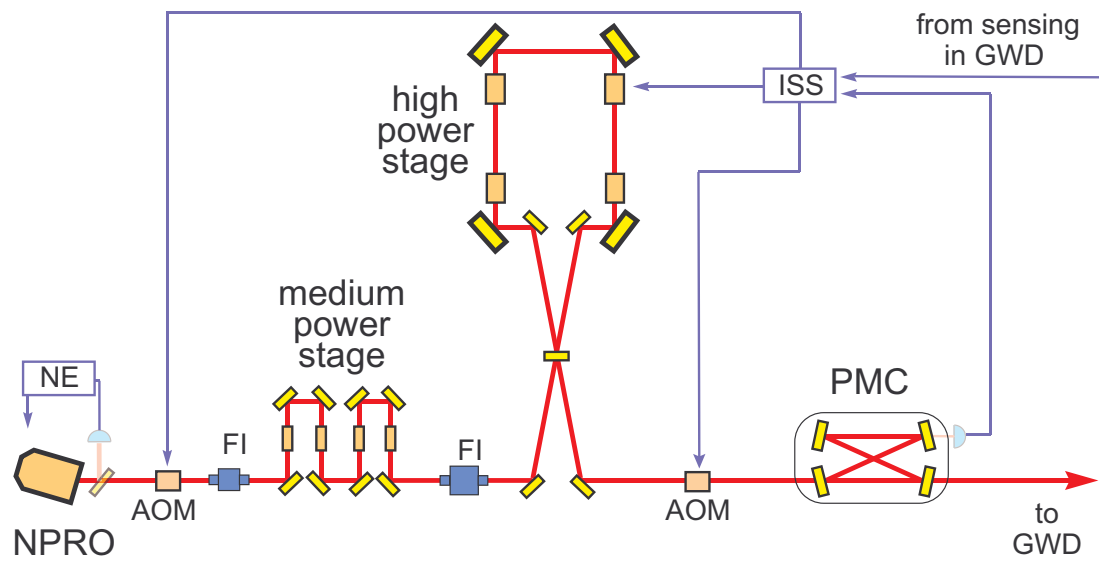

Figure 17 (online color at: www.lprjournal.org) Layout of the Advanced LIGO PSL system including a schematic of the nested power control scheme. AOM: acousto-optical modulator; FI: Faraday isolator; PMC: pre-mode-cleaner resonator; ISS: frequency stabilization electronics. 
such that power fluctuations caused by beam pointing or PMC length noise can be detected by this sensor. Several options exist to reduce the RPN at this point. The feedback signal could be sent to the AOM placed between the NPRO and the amplifier stage. This actuator has a high bandwidth of $100 \mathrm{kHz}$ but has a limited actuation range as the transfer function for relative power variations from the NPRO power to the power of the full system is $-20 \mathrm{~dB}$ up to $1 \mathrm{kHz}$. This means that we would have to vary the NPRO power with the AOM by 50\% to compensate for $5 \%$ relative power fluctuations on the $200 \mathrm{~W}$ beam. A second option is to modulate the pump diode power of the high-power stage. This actuator scheme has a wide range and reduces the noise in the high-power stage from where it most likely originates. The laser dynamics, however, reduces the useful bandwidth of this actuator, and a variation in the thermal lens is associated with the change in pump power. A third scheme would rely on an AOM placed into the $200 \mathrm{~W}$ beam. This actuator provides a large range and high bandwidth and would be the optimal choice, if it can withstand the high power on long time scales and does not introduce too large a spatial beam aberration.

A detailed study of the origin of the power noise in the $200 \mathrm{~W}$ laser and investigations of the performance of the high-power AOM are under way to design the final power stabilization control loop. This design will incorporate an input from an additional control loop filter to compensate for RPN sensed further downstream in the GWD where a stability level of RPN $\leq 2 \times 10^{-9} \mathrm{~Hz}^{-1 / 2}$ is required.

\section{Summary and outlook}

This paper presents a summary of the design of high-power laser systems to be used in GWDs. The detection principle of GWDs is explained and the design choices made for the laser system of the Advanced LIGO GWD are reviewed. The DBB serves as an example to explain fundamental laser characterization and stabilization techniques, and some measurements taken with the DBB are shown. Finally the frequency and power stabilization and spatial beam filtering with a PMC are discussed. Special emphasis is placed on power stabilization, as it sets the most demanding noise requirement on lasers for second-generation GWDs.

Design work on third-generation GWDs has already started, as for example in the design study for the Einstein Telescope (ET) funded by the European Union [74, 75]. These detectors will continue to set the most stringent requirements on the beam shape, temporal stability and reliability of their continuous-wave high-power light sources. Several new concepts in the laser and stabilization design are currently under investigation within the third working group of the ET design study. Fiber lasers show a promising performance and could replace the current solid-state lasers, and new sensing schemes will probably replace the classical RPN detection methods (e.g. [76,77]). It is possible that third-generation GWDs will require higher power levels and/or light of longer $(1550 \mathrm{~nm})$ or shorter $(532 \mathrm{~nm})$ wavelength. Hence it is expected that the research and development on GWD light sources will continue at a high level and that important contributions to future developments in the field of lasers and photonics will be made by this research.

Acknowledgements The high-power laser was developed and built by the Laserzentrum Hannover (Germany). I would like to thank the solid-state laser team at the Laserzentrum Hannover for providing the laser pictures and schematics of the $35 \mathrm{~W}$ and the $200 \mathrm{~W}$ laser systems. The work on laser stabilization was performed by the members of the Advanced LIGO PSL team from the Laserzentrum Hannover, the Max-Planck-Institut für Gravitationsphysik (Albert-Einstein-Institut), the Leibniz Universität Hannover and the LIGO Laboratory with financial support from the German Volkswagen Stiftung and from the United States National Science Foundation under contract number PHY-0107417. Additional support was given by the Deutsche Forschungsgemeinschaft as a part of Sonderforschungsbereich No. 407 and of the QUEST cluster of excellence. I would like to thank Albrecht Rüdiger and Peter King for their helpful comments during the preparation of this article.

Benno Willke received his Ph. D. de-
gree in physics in 1992 from the Uni-
versity of Hannover, Germany in the
field of plasma physics. In 1993 he
joined the GEO collaboration and
worked on the design and installation
of the GEO600 gravitational wave
detector. He spent one postdoctorial
year (1997-1998) in the Ginzton Lab-

\section{References}

[1] H. Grote (for the LIGO Scientific Collaboration), Class. Quantum Gravity 25, 114043 (2008).

[2] B. P. Abbott, R. Abbott, R. Adhikari, et al., Rep. Prog. Phys. 72, 076901 (2009).

[3] D. Tatsumi, R. Takahashi, K. Arai, et al., Class. Quantum Gravity 24, S399 (2007).

[4] F. Acernese, M. Alshourbagy, P. Amico, et al., Class. Quantum Gravity 25, 184001 (2008).

[5] M. A. Papa, Class. Quantum Gravity 25, 114009 (2008).

[6] B. Abbott et al. (for the LIGO Scientific Collaboration), and G. Santostasi, Astrophys. J. 683, L45 (2008).

[7] An updated list of LSC publications can be found at www.lsc-group.phys.uwm.edu/ppcomm/Papers. 
[8] P. Fritschel, Proc. SPIE - Int. Soc. Opt. Eng. 4856, 282 (2003).

[9] Virgo-Collaboration, Advanced Virgo Baseline Design, Virgo document number VIR-0027A-09 (Virgo documents can be accessed via the Virgo technical documentation system: https://tds.ego-gw.it/).

[10] G. Rempe, R. J. Thompson, H. J. Kimble, and R. Lalezari, Opt. Lett. 17, 363 (1992).

[11] B. Cimma, D. Forest, P. Ganau, B. Lagrange, J. M. Mackowski, C. Michel, J. L. Montorio, N. Morgado, R. Pignard, L. Pinard, and A. Remillieux, Appl. Opt. 45, 1436 (2006).

[12] F. Brückner, T. Clausnitzer, O. Burmeister, D. Friedrich, E. B. Kley, K. Danzmann, A. Tünnermann, and R. Schnabel, Opt. Lett. 33, 264 (2008).

[13] R. Abbott, R. Adhikari, G. Allen, S. Cowley, E. Daw, D. DeBra, J. Giaime, G. Hammond, M. Hammond, C. Hardham, J. How, W. Hua, W. Johnson, B. Lantz, K. Mason, R. Mittleman, J. Nichol, S. Richman, J. Rollins, D. Shoemaker, G. Stapfer, and R. Stebbins, Class. Quantum Gravity 19, 1591 (2002).

[14] G. Ballardin, L. Bracci, S. Braccini, C. Bradaschia, C. Casciano, G. Calamai, R. Cavalieri, R. Cecchi, G. Cella, E. Cuoco, E. D'Ambrosio, V. Dattilo, A. D. Virgilio, L. Fabbroni, F. Fidecaro, F. Frasconi, A. Gaddi, A. Gennai, G. Gennaro, A. Giazotto, G. Losurdo, L. Holloway, P. L. Penna, F. Lelli, E. Majorana, M. Mazzoni, F. Paoletti, M. Pasotti, A. Pasqualetti, R. Passaquieti, D. Passuello, R. Poggiani, P. Puppo, F. Raffaelli, P. Rapagnani, F. Ricci, P. Ruggi, R. Stanga, R. Taddei, F. Vetrano, A. Vicere, and Z. Zhang, Rev. Sci. Instrum. 72, 3643 (2001).

[15] A. Stochino, B. Abbot, Y. Aso, M. Barton, A. Bertolini, V. Boschi, D. Coyne, R. DeSalvo, C. Galli, Y. Huang, A. Ivanov, S. Marka, D. Ottaway, V. Sannibale, C. Vanni, H. Yamamoto, and S. Yoshida, Nucl. Instrum. Meth. A 598, 737 (2009)

[16] B. Willke, K. Danzmann, M. Frede, P. King, D. Kracht, P. Kwee, O. Puncken, R. L. S. (Jr), B. Schulz, F. Seifert, C. Veltkamp, S. Wagner, P. Wessels, and L. Winkelmann, Class. Quantum Gravity 25, 114040 (2008).

[17] W. J. Startin, M. A. Beilby, and P. R. Saulson, Rev. Sci. Instrum. 69, 3681 (1998).

[18] R. Nawrodt, A. Zimmer, T. Koettig, C. Schwarz, D. Heinert, M. Hudl, R. Neubert, M. Thürk, S. Nietzsche, W. Vodel, P. Seidel, and A. Tünnermann, J. Phys., Conf. Ser. 122, 012008 (2008).

[19] Y. Levin, Phys. Rev. D 57, 659 (1998).

[20] S. Rowan, J. Hough, and D. Crooks, Phys. Lett. A 347, 25 (2005).

[21] G. M. Harry, H. Armandula, E. Black, D. R. M. Crooks, G. Cagnoli, J. Hough, P. Murray, S. Reid, S. Rowan, P. Sneddon, M. M. Fejer, R. Route, and S. D. Penn, Appl. Opt. 45, 1569 (2006)

[22] I. Martin, H. Armandula, C. Comtet, M. M. Fejer, A. Gretarsson, G. Harry, J. Hough, J. M. M. Mackowski, I. MacLaren, C. Michel, J. L. Montorio, N. Morgado, R. Nawrodt, S. Penn, S. Reid, A. Remillieux, R. Route, S. Rowan, C. Schwarz, P. Seidel, W. Vodel, and A. Zimmer, Class. Quantum Gravity 25, 055005 (2008).

[23] V. B. Braginsky and F. Y. Khalili, Rev. Mod. Phys. 68, 1 (1996).
[24] T. Corbitt and N. Mavalvala, J. Opt. B, Quantum Semiclass. Opt. 6, S675 (2004)

[25] H. Vahlbruch, M. Mehmet, S. Chelkowski, B. Hage, A. Franzen, N. Lastzka, S. Gossler, K. Danzmann, and R. Schnabel, Phys. Rev. Lett. 100, 033602 (2008).

[26] H. Muller-Ebhardt, H. Rehbein, R. Schnabel, K. Danzmann, and Y. Chen, Phys. Rev. Lett. 100, 013601 (2008).

[27] S. Miyoki, T. Uchiyama, K. Yamamoto, H. Hayakawa, K. Kasahara, H. Ishitsuka, M. Ohashi, K. Kuroda, D. Tatsumi, S. Telada, M. Ando, T. Tomaru, T. Suzuki, N. Sato, T. Haruyama, Y. Higashi, Y. Saito, A. Yamamoto, T. Shintomi, A. Araya, S. Takemoto, T. Higashi, H. Momose, J. Akamatsu, and W. Morii, Class. Quantum Gravity 21, S1173 (2004).

[28] B. Abbott, et al. (for the LIGO Scientific Collaboration), New J. Phys. 11, 073032 (2009).

[29] E. Coccia, Physica B 280, 525 (2000).

[30] W. Wiechmann, T. Kane, D. Haserot, F. Adams, G. Truong, and J. Kmetec, in: Conference on Lasers and Electro-Optics (CLEO '98), San Francisco 1998, OSA Technical Digest Series, Vol. 6 (Optical Society of America, Washington, 1998), pp. 432-433.

[31] I. Zawischa, Injektionsgekoppelte diodengepumpte Nd:YAG- und Nd:YVO4-Laser für terrestrische interferometrische Gravitationswellendetektoren, $\mathrm{PhD}$ thesis, Universität Hannover, Germany (2003).

[32] J. R. Smith, J. Degallaix, A. Freise, H. Grote, M. Hewitson, S. Hild, H. Lück, K. A. Strain, and B. Willke, Class. Quantum Gravity 25, 035003 (2008).

[33] K. Somiya, Y. Chen, S. Kawamura, and N. Mio, Phys. Rev. D 73, 122005 (2006); Erratum: Phys. Rev. D 75, 049905 (2007).

[34] D. Sigg and N. Mavalvala, J. Opt. Soc. Am. A 17, 1642 (2000).

[35] P. Saulson, Fundamentals of Interferometric Gravitational Wave Detectors (World Scientific Publishing, Singapore, New Jersey, London, Hong Kong, 1994).

[36] T. Kane, J. Eggleston, and R. Byer, IEEE J. Quantum Electron. 21, 1195 (1985).

[37] Innolight, Mephisto product line (http://www.innolight.de/).

[38] B. Willke, S. Brozek, K. Danzmann, V. Quetschke, and S. Gossler, Opt. Lett. 25, 1019 (2000).

[39] P. Kwee and B. Willke, Appl. Opt. 47, 6022 (2008).

[40] W. Koechner, Appl. Opt. 9, 2548 (1970).

[41] T. Kane, R. Eckardt, and R. Byer, IEEE J. Quantum Electron. 19, 1351 (1983)

[42] J. Eggleston, T. Kane, K. Kuhn, J. Unternahrer, and R. Byer, IEEE J. Quantum Electron. 20, 289 (1984).

[43] A. Giesen, H. Hügel, A. Voss, K. Wittig, U. Brauch, and H. Opower, Appl. Phys. B, Lasers Opt. 58, 365 (1994).

[44] Y. Jeong, J. Nilsson, J. K. Sahu, D. S. Soh, C. Alegria, P. Dupriez, C. A. Codemard, D. N. Payne, R. Horley, L. M. B. Hickey, L. Wanzcyk, C. E. Chryssou, J. AlvarezChavez, and P. W. Turner, Conf. Lasers and Electro-Optics (CLEO), Tech. Dig. 2, 1065-1066 (2004).

[45] A. K. Sridharan, S. Saraf, S. Sinha, and R. L. Byer, Appl. Opt. 45, 3340 (2006).

[46] S. Tidwell, J. Seamans, M. Bowers, and A. Cousins, IEEE J. Quantum Electron. 28, 997 (1992). 
[47] M. P. Murdough and C. A. Denman, Appl. Opt. 35, 5925 (1996).

[48] M. Frede, Einfrequentes Laserlicht höchster Brillianz, PhD thesis, Leibniz Universität Hannover, Germany (2007).

[49] M. Frede, B. Schulz, R. Wilhelm, P. Kwee, F. Seifert, B. Willke, and D. Kracht, Opt. Express 15, 459 (2007).

[50] M. Frede, R. Wilheim, M. Brendel, C. Fallnich, F. Seifert, B. Willke, and K. Danzmann, Opt. Express 12, 3581 (2004).

[51] Q. Lü, N. Kugler, H. Weber, S. Dong, N. Müller, and U. Wittrock, Opt. Quantum Electron. 28, 57 (1996).

[52] M. Frede, R. Wilhelm, D. Kracht, and C. Fallnich, Opt. Express 13, 7516 (2005).

[53] B. Willke, N. Uehara, E. K. Gustafson, R. L. Byer, P. J. King, S. U. Seel, and R. L. Savage, Opt. Lett. 23, 1704 (1998).

[54] R. W. P. Drever, J. L. Hall, F. V. Kowalski, J. Hough, G. M. Ford, A. J. Munley, and H. Ward, Appl. Phys. B, Lasers Opt. 31, 97 (1983).

[55] E. D. Black, Am. J. Phys. 69, 79 (2001).

[56] A. Siegmann, Lasers (University Science Books, Mill Valley, California, 1986).

[57] A. Yariv, Optical Electronics in Modern Communications, 5th Edition (Oxford University Press, Oxford, 1997).

[58] E. Morrison, B. J. Meers, D. I. Robertson, and H. Ward, Appl. Opt. 33, 5041 (1994).

[59] M. Hildebrandt, M. Frede, P. Kwee, B. Willke, and D. Kracht, Opt. Express 14, 11071 (2006).

[60] P. Kwee, F. Seifert, B. Willke, and K. Danzmann, Rev. Sci. Instrum. 78, 073103 (2007).

[61] A. D. Ludlow, M. M. Boyd, T. Zelevinsky, S. M. Foreman, S. Blatt, M. Notcutt, T. Ido, and J. Ye, Phys. Rev. Lett. 96, 033003 (2006).

[62] H. Stoehr, F. Mensing, J. Helmcke, and U. Sterr, Opt. Lett. 31, 736 (2006).
[63] R. J. Rafac, B.C. Young, J. A. Beall, W. M. Itano, D. J. Wineland, and J.C. Bergquist, Phys. Rev. Lett. 85, $2462(2000)$.

[64] J. Ye, H. J. Kimble, and H. Katori, Science 320, 1734 (2008).

[65] F. Bondu, P. Fritschel, C. N. Man, and A. Brillet, Opt. Lett. 21, 582 (1996).

[66] A. D. Ludlow, X. Huang, M. Notcutt, T. Zanon-Willette, S. M. Foreman, M. M. Boyd, S. Blatt, and J. Ye, Opt. Lett. 32, 641 (2007).

[67] T. Nazarova, F. Riehle, and U. Sterr, Appl. Phys. B, Lasers Opt. 83, 531 (2006).

[68] K. Numata, A. Kemery, and J. Camp, Phys. Rev. Lett. 93, 250602 (2004).

[69] G. Harry, Workshop on optical coatings in precision measurements, Tech. Rep., Caltech, Pasadena, USA, (2008) (www.ligo.mit.edu/ gharry/workshop/booklet.pdf).

[70] J. Rollins, D. Ottaway, M. Zucker, R. Weiss, and R. Abbott, Opt. Lett. 29, 1876 (2004).

[71] B. W. Barr, K. A. Strain, and C. J. Killow, Class. Quantum Gravity 22, 4279 (2005).

[72] F. Seifert, P. Kwee, M. Heurs, B. Willke, and K. Danzmann, Opt. Lett. 31, 2000 (2006)

[73] P. Kwee, B. Willke, and K. Danzmann, Appl. Opt. 48, 5423 (2009).

[74] ET web page (http://www.et-gw.eu)

[75] S. Hild, S. Chelkowski, and A. Freise, arXiv:0810.0604v2 (gr-qc) (2008).

[76] P. Kwee, B. Willke, and K. Danzmann, Opt. Lett. 33, 1509 (2008).

[77] A. Khalaidovski, A. Thuring, H. Rehbein, N. Lastzka, B. Willke, K. Danzmann, and R. Schnabel, Phys. Rev. A 80, 053801 (2009). 